\title{
Ligustrazine ameliorates lipopolysaccharide-induced neurocognitive impairment by activating autophagy via the PI3K/AKT/mTOR pathway
}

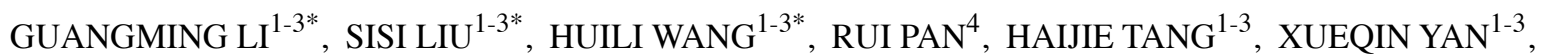 \\ YANPING WANG ${ }^{1-3}$, YONGMEI FU ${ }^{1-3}$, FUJUN JING ${ }^{1-3}$ and JUN DONG ${ }^{1-3}$ \\ ${ }^{1}$ Department of Pathophysiology, School of Medicine, Jinan University; ${ }^{2}$ Laboratory of Pathophysiology, \\ State Administration of Traditional Chinese Medicine, School of Medicine, Jinan University; ${ }^{3}$ GHM Institute of \\ CNS Regeneration, Jinan University; ${ }^{4}$ Department of Orthopedics, The First Affiliated Hospital, \\ Jinan University, Guangzhou, Guangdong 510632, P.R. China
}

Received November 4, 2019; Accepted February 17, 2020

DOI: $10.3892 /$ ijmm.2020.4548

\begin{abstract}
Autophagy is a lysosome-mediated cell contentdependent degradation pathway that leads to enhanced inflammation in an uncontrolled state. This study examined the role of autophagy in lipopolysaccharide (LPS)-induced brain inflammation and the effects of the traditional Chinese medicine ligustrazine on LPS-induced neurocognitive impairment in rats. Furthermore, the molecular mechanisms by which ligustrazine influences neurocognitive impairments were explored. The production of the inflammatory mediators interleukin (IL)-1 $\beta$ and tumor necrosis factor (TNF)- $\alpha$ was analyzed using ELISAs, and the expression levels of the autophagy marker microtubule-associated protein light chain 3 (LC3) II/I were analyzed using western blotting. LPS exposure upregulated the expression of IL-1 $\beta$ and TNF- $\alpha$ and downregulated the expression of LC3 II/I. Ligustrazine activated autophagy by preventing the expression of phosphoinositide 3-kinase (PI3K), phosphorylated protein kinase B (p-AKT), and phosphorylated mammalian target of rapamycin (p-mTOR). The present results suggest that ligustrazine improved LPS-induced neurocognitive impairments by activating autophagy and ameliorated neuronal injury by regulating the PI3K/AKT/mTOR signaling
\end{abstract}

Correspondence to: Professor Jun Dong, Laboratory of Pathophysiology, State Administration of Traditional Chinese Medicine, Jinan University, Room 1010 Administration building, 601 Huang Pu Avenue West, Guangzhou, Guangdong 510632, P.R. China

E-mail: dongjunbox@163.com

*Contributed equally

Abbreviations: LC3, lightchain3; PI3K, phosphatidylinositol 3 kinase; AKT, protein kinase $\mathrm{B}$; NF- $\mathrm{B}$, nuclear transcription factor- $\kappa \mathrm{B}$; mTOR, mammalian target of rapamycin

Key words: ligustrazine, lipopolysaccharide, neurocognitive impairment, inflammation, autophagy pathway. These findings provide an important reference for the prevention and treatment of neuroinflammation.

\section{Introduction}

With the aging of the global population and rapid increases in the size of the elderly population in China, there is an urgent need to develop interventions aimed at preventing neurocognitive disorders. Aging leads to neurotoxicity, resulting in the manifestation of cognitive dysfunction, which can affect abstract thinking, skilled movement, emotions, cognition and memory (1). Studies have found a strong link between inflammatory responses in certain regions of the central nervous system, a process termed 'neuroinflammation' and neurodegenerative diseases $(2,3)$. Primary neuronal injury may be the result of local neuroinflammation caused by the release of inflammatory mediators (4). The stereotaxic injection of lipopolysaccharide (LPS) is widely used to induce intracranial nerve inflammation as a model of neuroinflammation (5). LPS is a component of the cell wall of gram-negative bacteria, which prompts a number of different immune responses that result in brain inflammation to induce this model (6).

Traditional nonsteroidal anti-inflammatory drugs can have adverse effects as a result of their mechanism of action. Ligustrazine (TMP) is an amide alkaloid isolated from Ligusticum tubers and has been shown to dilate cerebrovascular vessels, decrease vascular resistance, increase blood flow to the brain and limbs, and improve microcirculation. TMP reduces platelet surface activity, inhibits platelet aggregation, and prevents thrombosis. Furthermore, TMP has anti-inflammatory and antioxidative effects (7). However, studies on the effects of TMP on neurocognitive impairments are lacking.

Certain neurodegenerative diseases are characterized by autophagy, a lysosome-mediated cell content-dependent degradation pathway. Autophagy is often involved in the pathological mechanism underlying certain diseases (8). For example, patients with neurodegenerative diseases such as Alzheimer's disease and Parkinson's disease exhibit accumulation of autophagosomes in neurons. Autophagy is known to affect 
aging and age-related diseases primarily through the regulation of metabolic pathways and food consumption. Autophagy and longevity are regulated by the insulin/insulin-like growth factor-1 signaling pathway, the mammalian target of rapamycin (mTOR) pathway, dietary restriction, and hunger (9). In numerous physiological and pathological settings, autophagy protects cells by promoting the degradation of excess or damaged cell components and promotes the circulation of amino acids, lipids, nutrients, and metabolites. Autophagy is the main pathway for the degradation of cytoplasmic proteins and organelles (10). Therefore, abnormal autophagy processes directly affect protein misfolding and accumulation, and the regulation of autophagy may be a key mechanism in the treatment of neurodegenerative diseases.

The authors' previous experiments demonstrated that LPS induces mild neurocognitive impairments in rats. The present study aimed to extend the previous findings by elucidating potential methods for the screening of neurodegenerative diseases to identify the pathway through which TMP improves LPS-induced neurocognitive impairments. The study also explored autophagy-related molecular mechanisms as well as the mechanisms underlying the prevention of neurodegenerative diseases.

\section{Materials and methods}

Animals. A total of 36 specific pathogen free (SPF) Sprague-Dawley (SD) rats (half male and female, $\sim 4-8$ weeks old, 120-180 g) were used in this study. The animals were provided by the Jinan Pengyue Experimental Animal Center (animal qualification certificate no. 3700920006246). The animals were raised in SPF conditions at the experimental animal center of Jinan University and were housed at a temperature of $22 \pm 2{ }^{\circ} \mathrm{C}$ and a relative humidity of $50 \pm 5 \%$ with a $12-\mathrm{h}$ light and dark cycle throughout the study. Food and water were provided ad libitum. Experiments were conducted following a 10-day quarantine period to allow for environmental habituation. Additionally, all experiments were conducted in accordance with the requirements of the experimental animal ethics committee of Jinan University and approved by them.

Behavioral training and water maze test. Place navigation experiments were used to assess learning and memory ability. Experiments were conducted over 6 days and the rats were subjected to the Morris water maze with a water depth of $\sim 26 \mathrm{~cm}$ (11). The water temperature during the experiments was $24 \pm 1^{\circ} \mathrm{C}$ and the maze platform was hidden beneath the water. The water maze was divided into four quadrants. On the first day, the rats were placed individually in the water in the N, E, S and W (N: North, E: East, S: South, W: West) quadrants in order, with their nose facing the wall. The time taken to find the hidden platform was recorded as the latency. The total search time was limited to $90 \mathrm{sec}$. If a rat failed to find the platform within the specified time, it was guided to swim to the platform and kept on the platform for $10 \mathrm{sec}$, and the latency was recorded as $90 \mathrm{sec}$. On the second day, the middle point of the E quadrant was used as the first entry point and the rats were placed individually in the pool in the N, E, S and $\mathrm{W}$ quadrants in order. This was repeated on days 3 to 6 and the latencies were recorded daily. Following the completion of water maze training, 36 SD rats were randomly divided into 6 groups ( $n=6$ for each group) based on the average escape time in the water maze on day 6 using a random number table.

Establishment of model groups by intraperitoneal injections of ligustrazine for assessing the preventative and treatment effects of ligustrazine on neurocognitive impairment. TMP hydrochloride for injection was obtained from Chengdu Beite Biotechnology (http://www.btyy.com/\#/). The animals were randomly divided into six groups ( $\mathrm{n}=6$ for each group) based on the water maze training results. The doses of TMP were based on preliminary results and previously published literature $(12,13)$, and 3 doses were used: 50, 100 and $200 \mathrm{mg} / \mathrm{kg}$. Injections were administered intraperitoneally daily for 5 days starting at $3 \mathrm{pm}$. Animals in groups 4, 5 and 6 were injected intraperitoneally with 50,100, and $200 \mathrm{mg} / \mathrm{kg}$ TMP $(1 \mathrm{ml} / 100 \mathrm{~g}$ body weight), respectively, whereas animals in groups 2 and 3 were treated with $0.9 \%$ normal saline $(1 \mathrm{ml} / 100 \mathrm{~g}$ body weight) in a similar manner. Rats in group 1 were used as the blank control.

Establishment of LPS-induced neurocognitive impairments. Except for the control group, all rats underwent lateral ventricle intubation and fixation using brain stereotaxic locator according to the manufacturer's protocol (RWD Life Science). For intracerebroventricular injections, 3\% pentobarbital sodium was injected intraperitoneally $(45 \mathrm{mg} / \mathrm{kg})$ as an anesthetic. Once the rats were adequately anesthetized, they were placed in a stereotaxic instrument. A razor was used to remove the fur. Iodine was applied as a disinfectant. An incision was made in the scalp along the midline using a blade. Based on a reference map, the following coordinates were used as the injection point: $1.0 \mathrm{~mm}$ from the anterior fontanelle, $1.8 \mathrm{~mm}$ (mediolateral) from the sagittal suture and $3.8 \mathrm{~mm}$ deep (dorsoventral). After identifying the injection point, a hole was drilled in the skull. Once the cerebrospinal fluid was visible, drilling was stopped and a cannula was inserted into the lateral ventricle and fixed with dental cement. After 3 days of recovery, lateral ventricle injections were performed. During drug administration, the rats were immobilized and a microinjection needle was vertically inserted into the cannula to a depth of 3.5-4.0 mm from the cerebral epidermis. For the sham group, $5 \mu \mathrm{l}$ artificial cerebrospinal fluid (Shanghai Canspec Scientific Instruments Co., Ltd.) was administered. The $150 \mu 1 / 5 \mu 1$ LPS was injected into the lateral ventricle in the model group, $50 \mathrm{mg} / \mathrm{kg}$ TMP group, $100 \mathrm{mg} / \mathrm{kg}$ TMP group and $200 \mathrm{mg} / \mathrm{kg}$ TMP group. Injection was paused for 2 min after the infusion of every $2 \mu \mathrm{l}$. The needle was left in place for $10 \mathrm{~min}$ at the end of the injection and then withdrawn slowly. Water maze testing commenced the following day.

Morris water maze and behavioral testing. Place navigation was performed as described above. For the spatial probe test, the rats were evaluated for their ability to remember the spatial location of the platform after locating the hidden platform. The time spent in the target quadrant and the number of times the animals crossed the target quadrant were measured. After the escape trial, the hidden platform was removed on the seventh day of the experiment. The quadrant diagonal to the platform quadrant was selected as the entry point. The number of times 
the animals crossed the platform area within $90 \mathrm{sec}$ and the time spent in the platform quadrant were recorded. However, an animal exposed to $200 \mathrm{mg} / \mathrm{kg}$ TMP plus LPS was found dead on day 3 of Morris water maze of behavioral testing. In addition, the remaining animals in $200 \mathrm{mg} / \mathrm{kg}$ TMP plus LPS group exhibited a worse mental state, which was characterized by drowsiness, apathy, a curled position in the home cage and disinterest in activities during the study.

Cytokine immunoassays. After $24 \mathrm{~h}$ of the completion of the behavioral tests, the remaining rats $(n=35)$ were sacrificed after anesthesia with pentobarbital sodium $(45 \mathrm{mg} / \mathrm{kg}$ ) by intraperitoneal injection and then the brain tissues were immediately collected using cervical decapitation. The cortex and hippocampus were dissected for further neurochemical analyses. The protein concentrations of interleukin (IL)- $1 \beta$ and tumor necrosis factor (TNF)- $\alpha$ in the brain were detected using rat-specific ELISA kits [IL-1 $\beta$, cat. no. SEA563Ra; TNF- $\alpha$, cat. no. SEA133Ra; Wuhan USCN Business Co., Ltd.]. Experimental procedures were performed in accordance with the manufacturer's protocols. Briefly, the brain tissues were rinsed in ice-cold PBS and the homogenates were centrifuged for $30 \mathrm{~min}$ at $5,000 \mathrm{x} \mathrm{g}$ at $4^{\circ} \mathrm{C}$. Then, $100 \mu \mathrm{l}$ resultant supernatant was added to a 96 -well plate coated with the appropriate purified antigen and incubated for $1 \mathrm{~h}$ at $37^{\circ} \mathrm{C}$. Absorption was measured at $450 \mathrm{~nm}$ using a microplate reader (Bio-Rad Laboratories, Inc.). The mean and standard error of triplicate measurements were calculated for each tested sample.

Western blot analysis. Brain tissues were treated with differing concentrations of compounds as previously indicated. The tissues were centrifuged once with PBS at $300 \mathrm{x}$ g for $5 \mathrm{~min}$ at $4^{\circ} \mathrm{C}$. Subsequently, the tissue samples were suspended in radioimmunoprecipitation assay buffer (Beyotime Institute of Biotechnology) containing $1 \%$ phenylmethanesulfonyl fluoride (Beyotime Institute of Biotechnology). The tissues were mechanically disrupted using steel balls and homogenized at $15 \mathrm{x} \mathrm{g}$ for $5 \mathrm{~min}$ in a homogenizer. The tissue homogenates were centrifuged at $4^{\circ} \mathrm{C}$ and $8,000 \mathrm{x}$ g for $15 \mathrm{~min}$ and the protein concentration was determined using a BCA kit (Beyotime Institute of Biotechnology), in which $60 \mu \mathrm{g}$ protein per sample was mixed with sample loading buffer and boiled for $5 \mathrm{~min}$. Samples were separated on an 8-15\% SDS-PAGE gel (Beyotime Institute of Biotechnology) at $80 \mathrm{~V}$ for $30 \mathrm{~min}$ followed by $120 \mathrm{~V}$ for $90 \mathrm{~min}$ in $1 \mathrm{X}$ running buffer and transferred to a polyvinylidene difluoride (PVDF) membrane (EMD Millipore). Each PVDF membrane was blocked in Tris-buffered saline/Tween (TBST) with 5\% nonfat milk or bovine serum albumin (BSA; Beyotime Institute of Biotechnology) for $60 \mathrm{~min}$ with gentle shaking at $25^{\circ} \mathrm{C}$ and then incubated with the designated primary antibodies $(1: 1,000)$ overnight at $4^{\circ} \mathrm{C}$. The primary antibodies were purchased from Cell Signaling Technology, Inc., [anti-AKT (cat. no. 4685), anti-PI3K (cat. no. 4257), anti-P62 (cat. no. 397949s), anti-Beclin1 (cat. no. 3495s), anti-NF- $\mathrm{B}$ (cat. no. 8242p), anti-LC3 (cat. no. 2775s), anti-p-AKT (cat. no. 13038p), anti-p-mTOR (cat. no. 5536), anti-mTOR (cat. no. 2983)]. Each PVDF membrane was washed three times in TBST for $10 \mathrm{~min}$ per wash and then incubated with the 1:5,000 goat anti-rabbit IgG (Beijing Ding guo Changsheng Biotechnology Co., Ltd.; cat. no. IA-0071) in TBST with 5\% nonfat milk or BSA for $1 \mathrm{~h}$ at $25^{\circ} \mathrm{C}$. The membranes were washed three times in TBST for 10 min per wash and then visualized by horseradish peroxidase (EMD Millipore; cat. no. 69078). The band intensities were quantified using Image-Pro Plus 6.0.

Statistical analysis. All experiments were repeated in triplicate. The data are presented as the means \pm standard errors of the mean. Statistical analyses were performed using SPSS 19.0 (IBM, Corps.). The data were statistically analyzed using repeated-measures ANOVA or one-way ANOVA followed by the Student-Newman-Keuls test or Bonferroni's T2 test. $\mathrm{P}<0.05$ was considered to indicate a statistically significant difference.

\section{Results}

Water maze and behavioral training results in SD rats. To rule out the effects of individual differences and genetic mutations on the swimming ability of SD rats, the rats were first trained to swim and remember the context of the water maze. Learning and memory were evaluated according to escape latency. The rats were trained for 6 days and calculated the average escape latency from the four quadrants over the last two days as the final escape latency. SD rats were randomly divided into six groups using a random number table $(n=6$ for each group). No significant differences in escape latency were observed between the groups $(\mathrm{P}>0.05$; Fig. 1$)$.

Effects of TMP on spatial learning and memory in rats with LPS-induced neurocognitive impairments. The results of the Morris water maze navigation experiment on days 0 (escape trial after training), 1, 3, 5 and 6 revealed that compared with the blank control and sham operation groups, the model group exhibited a significantly prolonged escape latency $(\mathrm{P}<0.05)$. Compared with that of the model group, the escape latency of the $50 \mathrm{mg} / \mathrm{kg}$ TMP group was significantly shorter $(\mathrm{P}<0.05$; Fig. 2A). The number of platform crossings and time spent in the target quadrant were significantly increased in the $50 \mathrm{mg} / \mathrm{kg}$ TMP group compared with the model group $(\mathrm{P}<0.05)$, suggesting that LPS-induced neurocognitive impairments were partly rescued in rats treated with TMP (Fig. 2B and C). On day 6, representative navigation paths revealed that the navigation strategy of the rats in the model group was poorer compared with that of the rats in the control group. The navigation strategy of the rats in the $50 \mathrm{mg} / \mathrm{kg}$ TMP group was improved compared with that of the rats in the model group (Fig. 2D). The results of the exploration experiment on day 7 revealed that the number of platform crossings and time spent in the target quadrant were reduced in the model group compared with the sham operation group, indicating that rats in the model group exhibited LPS-induced neurocognitive impairments.

Expression of inflammatory factors underlying LPS-induced neurocognitive impairments. Compared with those in the sham operation group, the cortical and hippocampal IL-1 $\beta$ and TNF- $\alpha$ levels in the LPS treatment group were elevated $(\mathrm{P}<0.05)$, suggesting that LPS induced neuroinflammation in rats that resulted in neurocognitive impairments. Compared with those in the model group, the levels of IL- $1 \beta$ and TNF- $\alpha$ 


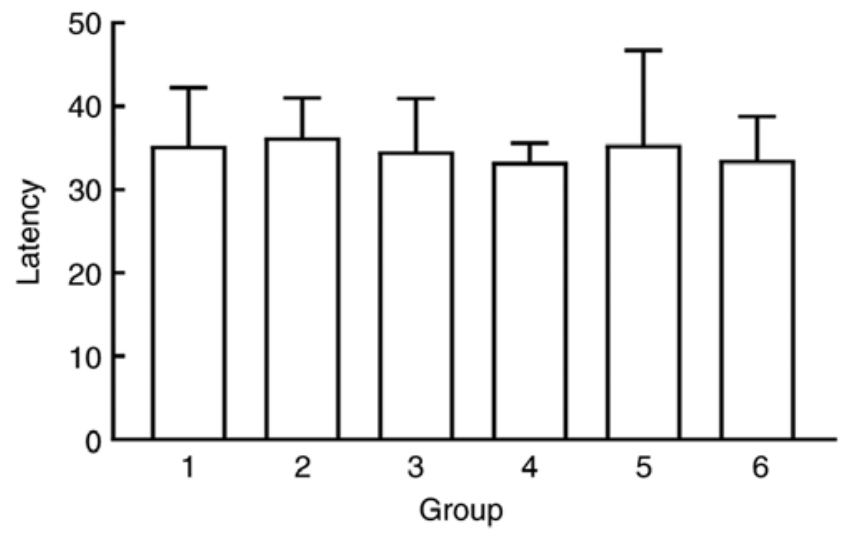

Figure 1. After water maze training, the animals are randomly assigned to the groups according to the escape latency (group 1: Blank control group, group 2: Sham operation group, group 3: Model group, group 4: $50 \mathrm{mg} / \mathrm{kg}$ TMP group, group 5: $100 \mathrm{mg} / \mathrm{kg}$ TMP group and group 6: $200 \mathrm{mg} / \mathrm{kg}$ TMP group). TMP, ligustrazine.

in the cortex and hippocampus of the $50 \mathrm{mg} / \mathrm{kg}$ TMP group were decreased $(\mathrm{P}<0.05)$. There were no significant differences between the $100 \mathrm{mg} / \mathrm{kg}$ TMP and $200 \mathrm{mg} / \mathrm{kg}$ TMP groups $(\mathrm{P}>0.05)$, suggesting that low-dose TMP ameliorated LPS-induced neuroinflammatory responses and improved neurocognitive impairments (Fig. 3).

Effects of TMP on LPS-induced cognitive impairments involve autophagy-related protein expression. Autophagy-related protein expression was analyzed by western blotting (Fig. 4A). Compared with the sham group, the model group exhibited significantly decreased LC3 II/I expression $(\mathrm{P}<0.05)$. Compared with the model group, the $50 \mathrm{mg} / \mathrm{kg}$ TMP group showed significantly increased LC3 II/I expression $(\mathrm{P}<0.05$; Fig. 4B). Compared with that in the sham group, p62 expression in the model group was significantly increased $(\mathrm{P}<0.05)$. Compared with that in the model group, p62 expression in the $50 \mathrm{mg} / \mathrm{kg}$ TMP group was reduced ( $\mathrm{P}<0.05$; Fig. 4C). Beclin1 expression in the model group was significantly decreased compared with that in the sham group $(\mathrm{P}<0.05)$, and Beclin1 expression in the $50 \mathrm{mg} / \mathrm{kg}$ TMP group was significantly increased $(\mathrm{P}<0.05$; Fig. 4D).

TMP improves LPS-induced neurocognitive impairments by activating autophagy through the PI3K/AKT/mTOR signaling pathway. The expression levels of p-mTOR, mTOR, p-AKT, AKT, PI3K and NF- $\kappa \mathrm{B}$ were analyzed in the blank control group, sham operation group, model group, and $50 \mathrm{mg} / \mathrm{kg}$ TMP group (Fig. 5A). Compared with that in the sham group, $\mathrm{p}-\mathrm{mTOR} / \mathrm{mTOR}$ expression in the model group was significantly increased $(\mathrm{P}<0.05)$. Compared with the model group, the $50 \mathrm{mg} / \mathrm{kg}$ TMP group showed significantly reduced $\mathrm{p}$-mTOR/mTOR expression $(\mathrm{P}<0.05)$. $\mathrm{p}$-AKT/AKT expression was significantly increased in the model group compared with the sham group $(\mathrm{P}<0.05$; Fig. 5B). There was a significant difference in the reduction in $\mathrm{p}-\mathrm{AKT} / \mathrm{AKT}$ expression in the $50 \mathrm{mg} / \mathrm{kg}$ TMP group compared with the model group $(\mathrm{P}<0.05$; Fig. 5C). There was a significant increase in PI3K levels in the model group compared with the sham group $(\mathrm{P}<0.05)$ and a significant decrease in the low-dose group compared with the model group ( $\mathrm{P}<0.05$; Fig. 5D). Additionally, there were significant increases in $\mathrm{NF}-\kappa \mathrm{B}$ levels in the model group compared to the sham group $(\mathrm{P}<0.05)$. However, there were no significant differences in NF- $\kappa \mathrm{B}$ levels between the model and $50 \mathrm{mg} / \mathrm{kg}$ TMP groups (P>0.05; Fig. 5E).

\section{Discussion}

More research on the pharmacological effects of traditional Chinese medicine may result in the discovery of novel drugs and molecular mechanisms that may provide new opportunities for the prevention and treatment of neurocognitive disorders. The behavioral tests used in this study revealed that $50 \mathrm{mg} / \mathrm{kg}$ TMP improved learning and memory in LPS-treated rats. Similar to the results described by Bai et al (14), it was observed that TMP reduced neuroinflammation caused by cerebral ischemia/reperfusion injury and improved overall outcomes following brain injury. Additionally, this study showed that TMP was protective against neurocognitive impairments.

Recovery from neurocognitive impairments may be attributed to the inhibition of neuroinflammation in the brain, the relief of neuronal edema and increased central blood circulation. In this study, IL- $1 \beta$ and TNF- $\alpha$ levels in the cortex and hippocampus of the rats in the model group were increased, indicating the occurrence of neuroinflammation in this group. In rats treated with $50 \mathrm{mg} / \mathrm{kg}$ TMP, IL- $1 \beta$ and TNF- $\alpha$ levels in the cortex and hippocampus were reduced, suggesting that $50 \mathrm{mg} / \mathrm{kg}$ TMP inhibited neuroinflammatory processes in the brain, thereby improving neurocognitive impairments. The present study found that IL- $1 \beta$ and TNF- $\alpha$ were not significantly reduced in the $100 \mathrm{mg} / \mathrm{kg}$ TMP and $200 \mathrm{mg} / \mathrm{kg}$ TMP groups following 5 days of ligustrazine pretreatment compared with the blank control group. Additionally, when compared with that of the model group, the escape latency of the $100 \mathrm{mg} / \mathrm{kg}$ TMP and $200 \mathrm{mg} / \mathrm{kg}$ TMP groups was not shorter. These findings indicate that neurocognitive impairments in the $100 \mathrm{mg} / \mathrm{kg}$ TMP and $200 \mathrm{mg} / \mathrm{kg}$ TMP rats were not significantly improved after ligustrazine pretreatment; therefore, $100 \mathrm{mg} / \mathrm{kg}$ TMP and $200 \mathrm{mg} / \mathrm{kg}$ TMP were considered to have had no effect.

LC3 attaches to the membrane surface of both preautophagic and autophagic vesicles and is involved in the formation of autophagosomes. LC3 expression is regarded as a marker of the degree of autophagy activation and for the diagnosis of altered autophagy (15). LC3 II is a derivative of LC3 I formed by ubiquitin-proteasome proteolysis after processing during autophagy and the formation of autophagic bodies. Compared with the model group, the $50 \mathrm{mg} / \mathrm{kg}$ TMP group exhibited increased LC3 II/I levels, suggesting that TMP activated autophagy. Changes in autophagy activity can be measured by observing changes in the expression of the autophagy-specific degradation substrate p62 since p62 plays an important role as a selective adaptor protein in autophagy. The PB1 domain of p62 promotes the packaging of ubiquitination substrates through oligomerization and transports packaged substrates to participate in the formation of autophagosomes (16). The p62 complex is formed through the interaction between the LIR domain of p62 and LC3, and is degraded as an autophagy-specific substrate in autophagic lysosomes (17). This study found that in the $50 \mathrm{mg} / \mathrm{kg}$ TMP 

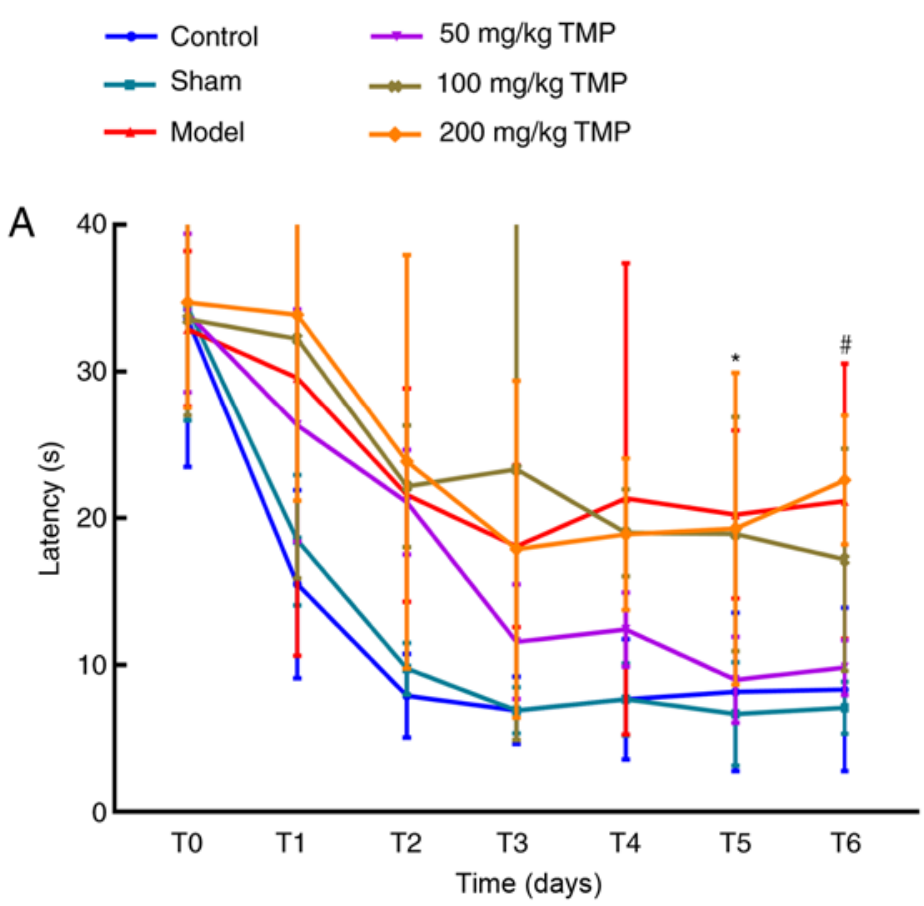
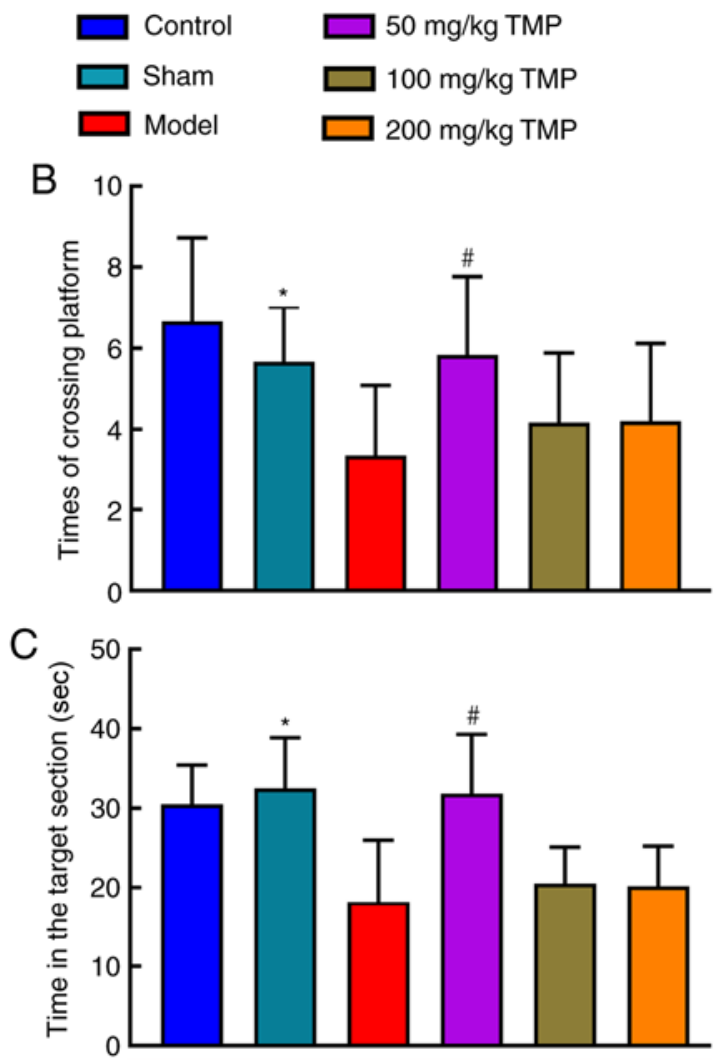
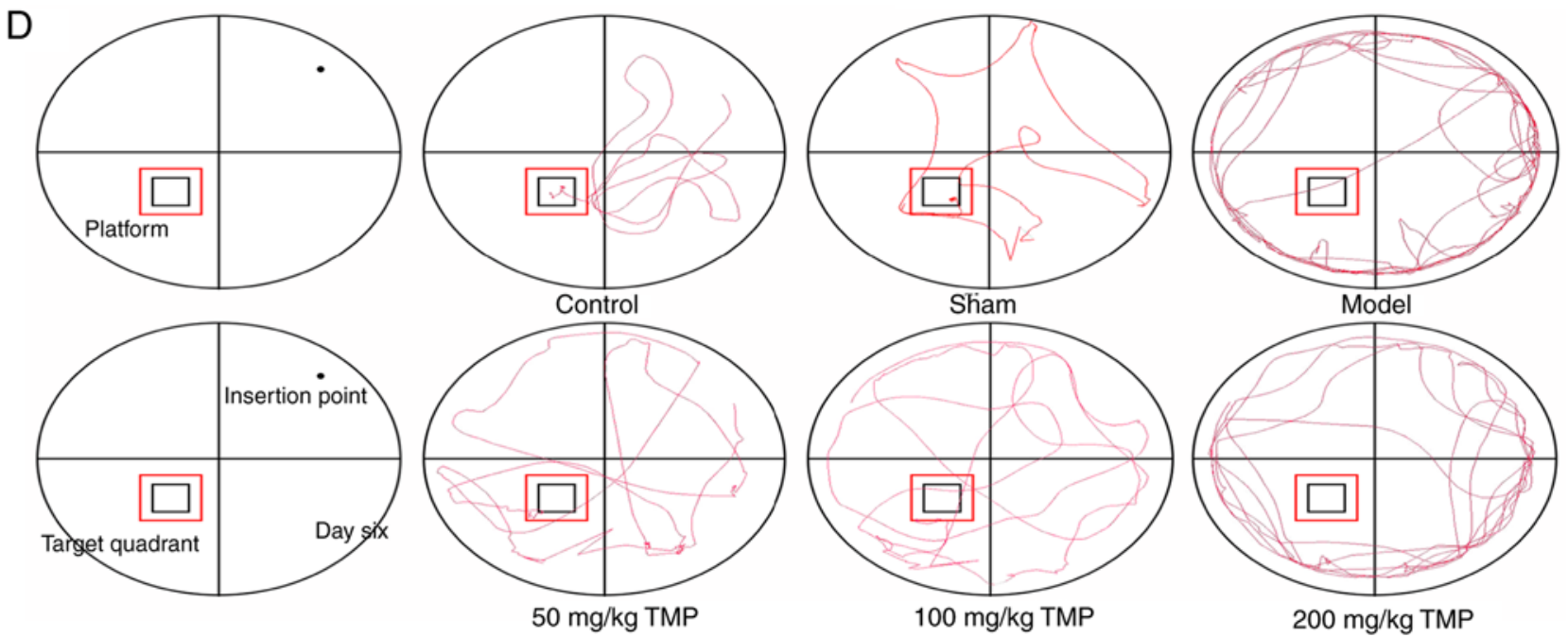

Swimming paths

Figure 2. Ligustrazine hydrochloride improves the spatial learning and memory of SD rats after LPS treatment. (A) The water maze escape latencies of SD rats intracerebroventricularly injected with artificial cerebrospinal fluid or LPS; latencies on the fifth and sixth days, representing spatial learning and recognition, are depicted. The spatial probe test was used to measure of the (B) number of crossings of and the (C) time spent in the target platform location after treatment with ligustrazine hydrochloride. On the final day, a 90 -sec probe trial was conducted to detect the number of times the animals entered the small target zone and the time they spent in the target quadrant. (D) Representative swimming paths during exploration on day 6 of training. The data are presented as the mean \pm SEM, groups 1 to $5: \mathrm{n}=6$, group $6: \mathrm{n}=5$. ${ }^{\text {}} \mathrm{P}<0.05$, the $50 \mathrm{mg} / \mathrm{kg}$ TMP group vs. the model group; ${ }^{*} \mathrm{P}<0.01$, the model group vs. the sham group. $\mathrm{SD}$ rats, Sprague-Dawley rats; LPS, lipopolysaccharide; TMP, ligustrazine.

group, which showed increased LC3 II/I levels, the accumulation of p62 was reduced, suggesting the activation of autophagy in the brain. Beclin1 is a key molecule in autophagy that mediates the recruitment of autophagy-related proteins to autophagic vesicles and promotes the formation and maturation of autophagosomes by binding various proteins. Beclin1 regulates autophagy alongside various factors that regulate mammalian class III PI3K (Vps34). The regulation of the synthesis of the Beclin1 Vps34-Vps15 core complex can alter autophagy activity (18). The results of this study showed that compared with the model group, the $50 \mathrm{mg} / \mathrm{kg}$ TMP group exhibited increased Beclin1 synthesis following the activation of autophagy. These results demonstrated that TMP can activate autophagy and improve neurocognitive impairment. Interestingly, the current study found that compared with the animals in the $50 \mathrm{mg} / \mathrm{kg}$ TMP group, the animals in the 
A
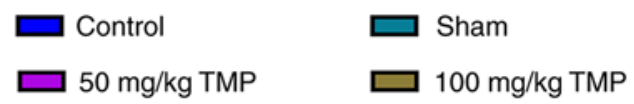

$\square$ Model

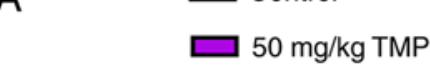

$\square 200 \mathrm{mg} / \mathrm{kg}$ TMP
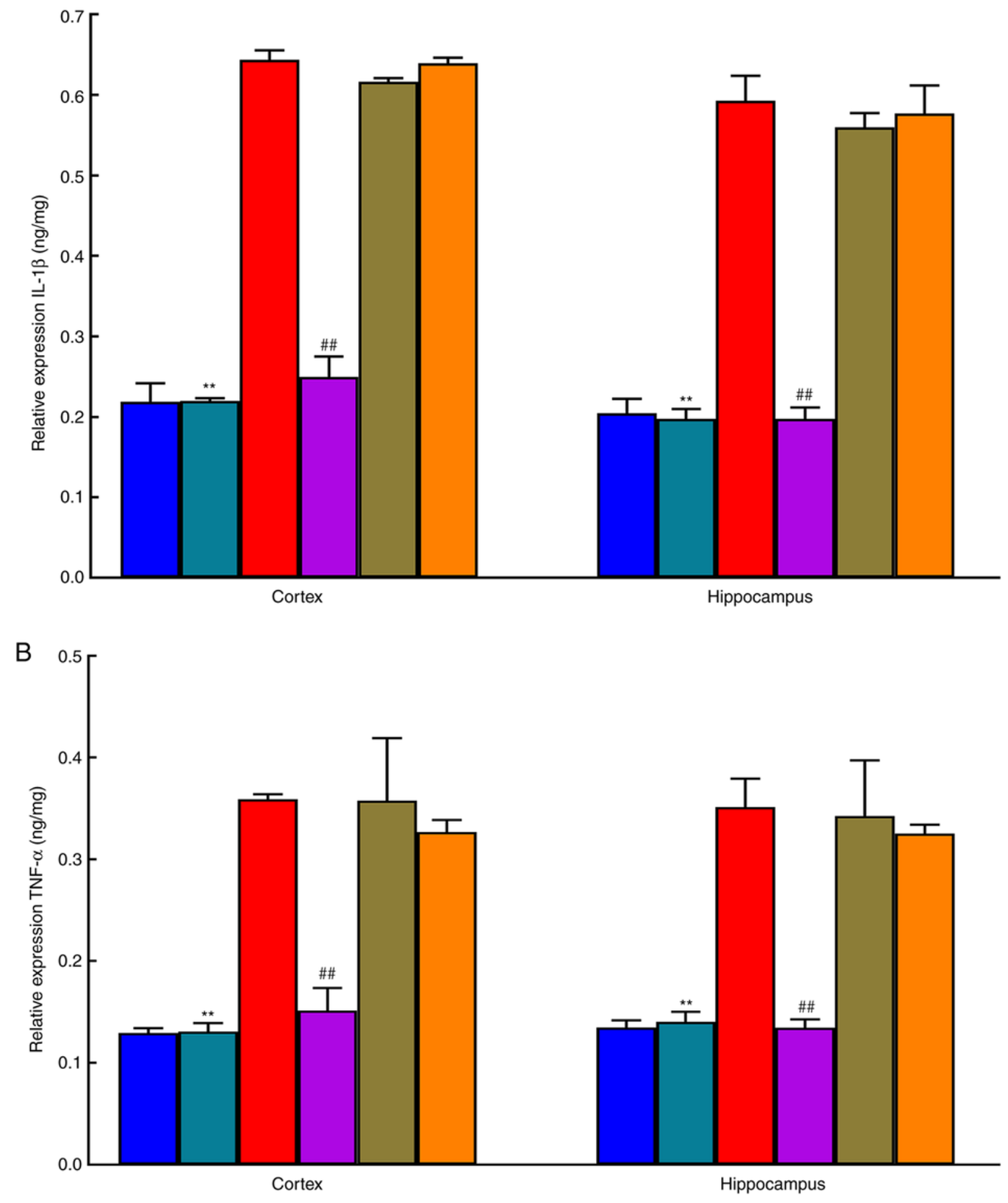

Figure 3. Ligustrazine improves LPS-induced neurocognitive impairments based on the relative expression levels of inflammatory factors. (A) The relative expression levels of IL-1 $\beta$ in the cortex and hippocampus, as measured by ELISA. (B) The relative expression levels of TNF- $\alpha$ in the cortex and hippocampus, as measured by ELISA. The data are presented as the mean \pm SEM, $\mathrm{n}=3$. ${ }^{\# \#} \mathrm{P}<0.01$, the $50 \mathrm{mg} / \mathrm{kg}$ TMP group vs. the model group; ${ }^{* *} \mathrm{P}<0.01$, the model group vs. the sham group. LPS, lipopolysaccharide; IL, interleukin; TNF, tumor necrosis factor; TMP, ligustrazine.

$200 \mathrm{mg} / \mathrm{kg}$ TMP group exhibited a worse mental state characterized by drowsiness, apathy, a curled position in the home cage and disinterest in activities following the injection of ligustrazine. In addition, an animal was found dead with a stiff and cold body in the $200 \mathrm{mg} / \mathrm{kg}$ TMP group. No abnormal changes were observed by the anatomy analysis. Combined with the poor state of animals exposed to $200 \mathrm{mg} / \mathrm{kg}$ TMP plus LPS, it is considered that the synergistic effect of $200 \mathrm{mg} / \mathrm{kg}$ TMP and LPS may be the reason. However, the exact cause is unclear and will be investigated further. Taken together, these 

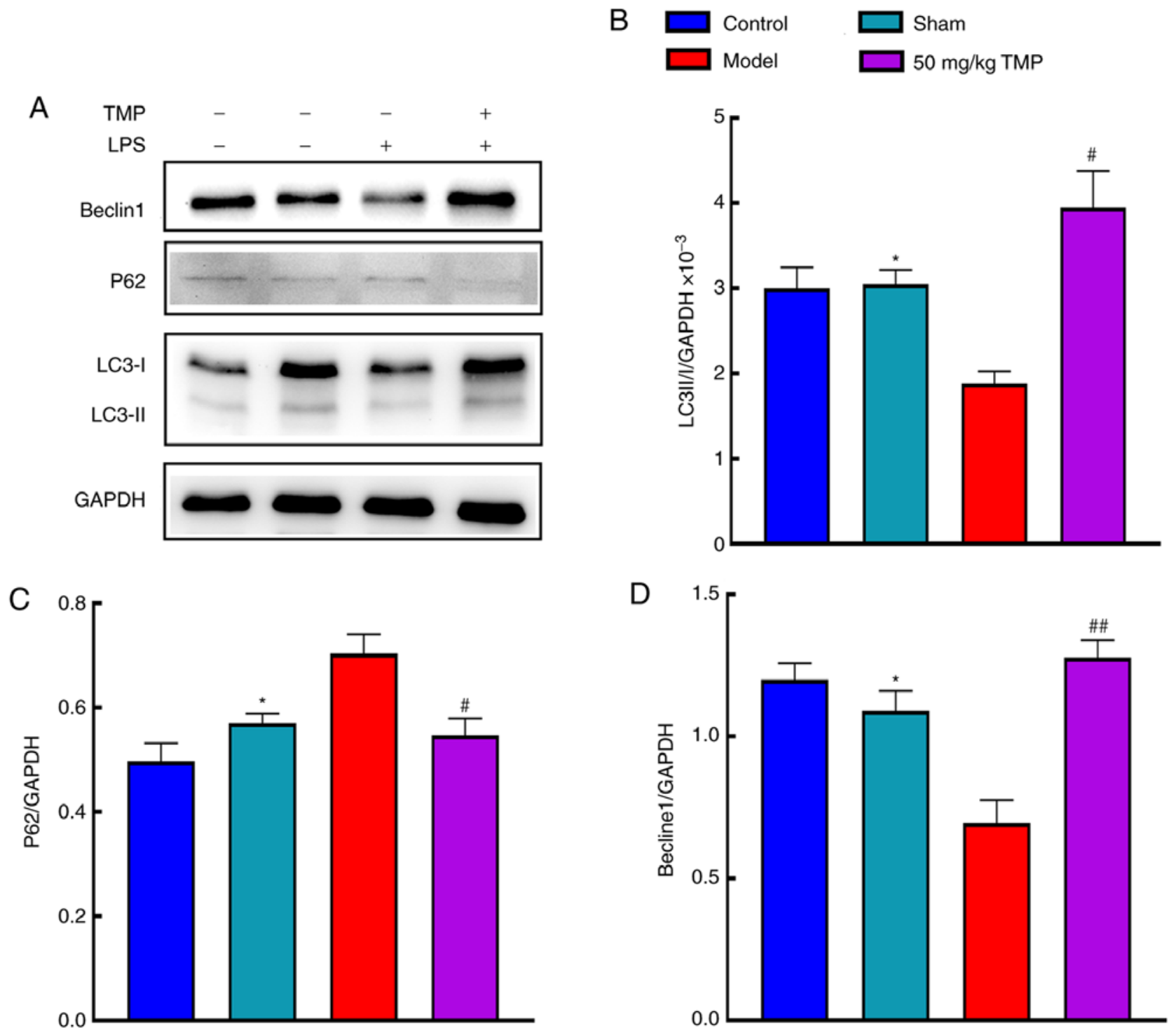

Figure 4. Ligustrazine hydrochloride improves the protein expression levels of LC3 II/I, p62 and Beclin1 in the brain of SD rats after LPS treatment. (A) The protein expression levels of LC3 II/I, p62 and Beclin1 were determined by western blot analysis. (B) Ligustrazine hydrochloride improved the protein expression level of LC3II/I in the brain of SD rats after LPS treatment. (C) Ligustrazine hydrochloride improved the protein expression level of p62 in the brain of SD rats after LPS treatment. (D) Ligustrazine hydrochloride improved the protein expression level of Beclin1 in the brain of SD rats after LPS treatment. The data are presented as the mean \pm SEM, $n=3 .{ }^{*} \mathrm{P}<0.05$ and ${ }^{\# \#} \mathrm{P}<0.01$, the $50 \mathrm{mg} / \mathrm{kg}$ TMP group vs. the model group; ${ }^{*} \mathrm{P}<0.05$, the model group vs. the sham group. LC3, light chain 3; SD, Sprague-Dawley; LPS, lipopolysaccharide; TMP, ligustrazine.

findings, along with the subsequent behavioral evaluations and detection of inflammatory markers, support the hypothesis that $50 \mathrm{mg} / \mathrm{kg}$ doses of ligustrazine can induce a significant pharmacological effect with respect to improving neurocognitive functions.

Although the specific mechanisms of neuronal autophagy are not fully understood, the $\mathrm{PI} 3 \mathrm{~K} / \mathrm{AKT} / \mathrm{mTOR}$ pathway is a classical upstream activation pathway of autophagy (19). It is generally believed that receptor tyrosine kinases, such as transmembrane glycoproteins, can react with relevant extracellular ligands. The activation of tyrosine residues of receptor tyrosine kinase recruits the p85 regulatory subunit to PI3K to activate the p110 catalytic subunit. Phosphatidylinositol 4-phosphate and phosphatidylinositol 3,4-bisphosphate (PIP2) have a hydroxyl group on the inositol ring, which is activated by PI3K phosphorylation; this produces PIP2 and 3,4,5-inositol (PIP3) independently. PIP3 is a second messenger and an important molecule involved in PI3K signal transmission, as it recruits signaling molecules of the $\mathrm{PH}$ domain to the plasma membrane for activation (20).

The core structure of AKT is composed of three parts: The regulatory active region at the $\mathrm{C}$ terminus, the catalytic active region in the middle and the $\mathrm{PH}$ domain at the $\mathrm{N}$ terminus. The PH domain of AKT is phosphorylated by activated PI3K. AKT has catalytic activity at Thr308 and regulatory activity at Ser473. When phosphoinositol-dependent kinases (PDK)1 and PDK2 participate in the reaction, AKT is phosphorylated at Ser473 and Thr308, thereby activating and phosphorylating AKT to produce $\mathrm{p}-\mathrm{AKT}$, which results in various physiological effects. Activated p-AKT phosphorylates mTOR directly (21). Mammalian mTOR is a component of mechanistic target of rapamycin $\mathrm{C} 1$ (mTORC1). When it is sensitive to rapamycin, it regulates intracellular nutrition, growth factors, pressure and other signals and activates PRAS40, a protein that binds 

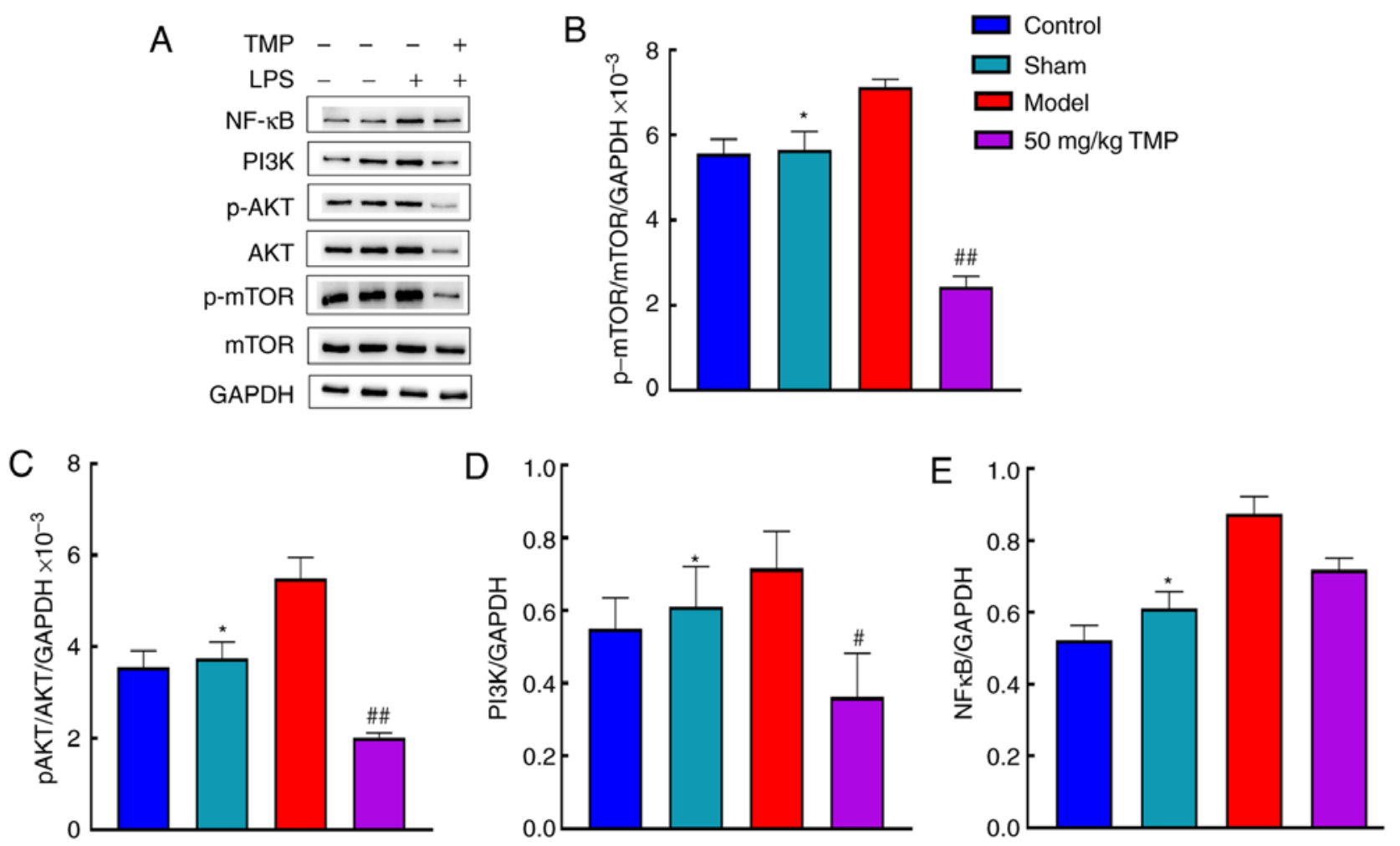

Figure 5. Ligustrazine hydrochloride improves the protein expression levels of NFKB, PI3K, p-AKT, AKT, p-mTOR and mTOR in the brain of SD rats after LPS treatment. (A) The protein expression levels of NFKB, PI3K, p-AKT, AKT, p-mTOR and mTOR were determined by western blot analysis. (B) Ligustrazine hydrochloride improved the protein expression level of p-mTOR/mTOR in the brain of SD rats after LPS treatment. (C) Ligustrazine hydrochloride improved the protein expression level of (C) p-AKT/AKT, (D) PI3K and (E) NFאB in the brain of SD rats after LPS treatment. The data are presented as the mean \pm SEM, $\mathrm{n}=3 .{ }^{*} \mathrm{P}<0.05$ and ${ }^{\# \#} \mathrm{P}<0.01$ for $50 \mathrm{mg} / \mathrm{kg}$ TMP vs. model group; ${ }^{*} \mathrm{P}<0.05$ for model group vs. sham. NF- $\mathrm{B}$, nuclear transcription factor- $\mathrm{kB}$; PI3K, phosphatidylinositol 3 kinase; p-AKT, protein kinase B; SD, Sprague-Dawley; LPS, lipopolysaccharide; mTOR, mammalian target of rapamycin; TMP, ligustrazine.

to mTORC1, causing PRAS40 to separate from mTORC1 (22). When it is not sensitive to rapamycin, it controls cell proliferation and survival. When energy supplies are impaired or the oxygen level is insufficient, Raptor, a component of mTORC1, is phosphorylated. Phosphorylated Raptor inhibits the activity of mTORC1, while phosphorylation activates the downstream unc-5 autophagy activated kinase (ULK) complex (including ulk1/2 and Atg13), increasing the activation of ulk1/2 and the phosphorylation level of Atg13. Once ULK1 is activated, it forms a complex with Beclin1, leading to the activation and binding of Beclin1 with the autophagy-related protein Atg1-Atg13 complex. This promotes Atg1 activation and induces autophagy initiation or upregulation to maintain cell homeostasis $(23,24)$. The results of this study showed that compared with the sham operation group, the model group showed increased PI3K, p-AKT/AKT and p-mTOR/mTOR, suggesting that the inhibition of autophagy in the model group may have been mediated by the PI3K/AKT/mTOR signaling pathway. Compared with the levels in the model group, the PI3K, p-AKT/AKT and $\mathrm{p}$-mTOR/mTOR levels were decreased in the $50 \mathrm{mg} / \mathrm{kg}$ TMP group, indicating that autophagy was activated in the $50 \mathrm{mg} / \mathrm{kg}$ TMP group, possibly through the PI3K/AKT/mTOR signaling pathway. Studies have shown that various external factors can activate the NF- $\kappa \mathrm{B}$ signaling pathway, including cellular stress, ionizing radiation, LPS, cellular membrane proteins and virus-related membrane proteins $(25,26)$. The NF- $\mathrm{NB}$ signaling pathway is an important pathway that regulates inflammatory responses. Studies have shown that icariin activates autophagy by inhibiting the expression of NF- $\kappa \mathrm{B}$ signaling during chondrocyte apoptosis $(27,28)$. However, the present study found that the expression levels of IL- $1 \beta, \mathrm{TNF}-\alpha$ and NF- $\mathrm{BB}$ were increased in the model group compared with the sham group, suggesting that LPS stimulation promoted the expression of $\mathrm{NF}-\kappa \mathrm{B}$ and induced inflammation. However, compared with the model group, the $50 \mathrm{mg} / \mathrm{kg}$ TMP group showed no significant difference in the protein expression level of NF- $\kappa \mathrm{B}$, suggesting that the activation of autophagy in the $50 \mathrm{mg} / \mathrm{kg}$ TMP group did not affect the NF- $\kappa \mathrm{B}$ signaling pathway. Autophagy activation in the $50 \mathrm{mg} / \mathrm{kg}$ TMP group may have improved neurocognitive impairments through the PI3K/AKT/mTOR signaling pathway rather than by reducing inflammatory responses and inhibiting the expression of the NF- $\kappa \mathrm{B}$ protein. In this study, it was found that TMP activated autophagy, not through the NF- $\kappa \mathrm{B}$ pathway, but by inhibiting the PI3K/AKT/mTOR pathway, which led to reduced neuroinflammation and an improvement in neurocognition. However, the specific mechanisms underlying these effects remain unclear and should be clarified in future studies.

In conclusion, TMP improves LPS-induced neurocognitive impairments in rats. TMP may activate autophagy through the $\mathrm{PI} 3 \mathrm{~K} / \mathrm{AKT} / \mathrm{mTOR}$ signaling pathway, thereby improving the inflammatory responses of neurons, increasing learning and memory, and improving neurocognitive impairments in rats.

\section{Acknowledgements}

Not applicable. 


\section{Funding}

The present study was supported by grants from the National Natural Science Foundation of China (grant nos. 81974185 and 81471235), the Guangdong Provincial Natural Science Foundation of China (grant no. 2019A1515012024), the Program of Introducing Talents of Discipline to Universities (grant no. B14036), the Science and Technology Foundation of Guangdong (grant no. 2010B030700016), the Cultivation and Innovation Fund of Jinan University (grant no. 21617460), and the Medical Group Fund of Jinan University (grant no. 88016013039 ).

\section{Availability of data and materials}

The data are available from the corresponding author upon reasonable request.

\section{Authors' contributions}

GL and JD designed the study and wrote the initial draft of the manuscript. SL contributed to the analysis and interpretation of data and assisted in the preparation of the manuscript. HW contributed to data collection, and interpretation and critically reviewed the manuscript. YW participated in and performed the experiments in this study. YF supervised the methods of all the experiments and interpreted of results. HT provided help for analyzing the data. XY directed the study implementation, including quality assurance and control of experiments. RP and FJ supervised the present study and revised the manuscript. GL and JD revised the manuscript. All authors approved the final version of the manuscript and agreed to be accountable for all aspects of the work in ensuring that questions related to the accuracy or integrity of any part of the work are appropriately investigated and resolved.

\section{Ethics approval and consent to participate}

All procedures performed in studies involving animals were in accordance with the ethical standards of the institution or practice at which the studies were conducted (Experimental Animal Ethics Committee of Jinan University; Approval no. I ACUC-20180604-01).

\section{Patient consent for publication}

Not applicable.

\section{Competing interests}

The authors declare that they have no competing interests. The founding sponsors had no role in the design of the study; in the collection, analyses, or interpretation of the data; in the writing of the manuscript; or in the decision to publish the results.

\section{References}

1. Kanchi PK and Dasmahapatra AK: Polyproline chains destabilize the Alzheimer's amyloid-beta protofibrils: A molecular dynamics simulation study. J Mol Graph Model 93: 107456, 2019.
2. Leitner GR, Wenzel TJ, Marshall N, Gates EJ and Klegeris A: Targeting toll-like receptor 4 to modulate neuroinflammation in central nervous system disorders. Expert Opin Ther Targets 23: 865-882, 2019.

3. Ransohoff RM: How neuroinflammation contributes to neurodegeneration. Science 353: 777-783, 2016.

4. Augusto-Oliveira M, Arrifano GP, Lopes-Araújo A, SantosSacramento L, Takeda PY, Anthony DC, Malva JO and Crespo-Lopez ME: What do microglia really do in healthy adult brain? Cells 8: pii: E1293, 2019.

5. Batista CRA, Gomes GF, Candelario-Jalil E, Fiebich BL and de Oliveira ACP: Lipopolysaccharide-induced neuroinflammation as a bridge to understand neurodegeneration. Int $\mathrm{J}$ Mol Sci 20: pii: E2293, 2019

6. Li DW, Zhou FZ, Sun XC, Li SC, Yang JB, Sun HH and Wang AH: Ginsenoside $\mathrm{Rb} 1$ protects dopaminergic neurons from inflammatory injury induced by intranigral lipopolysaccharide injection. Neural Regen Res 14: 1814-1822, 2019.

7. Gong X, Ivanov VN, Davidson MM and Hei TK: Tetramethylpyrazine (TMP) protects against sodium arsenite-induced nephrotoxicity by suppressing ROS production, mitochondrial dysfunction, pro-inflammatory signaling pathways and programed cell death. Arch Toxicol 89: 1057-1070, 2015.

8. Yang L, Wang H, Liu L and Xie A: The role of insulin/IGF-1/ PI3K/Akt/ GSK3 $\beta$ signaling in parkinson's disease dementia. Front Neurosci 12: 73, 2018.

9. Suzuki H, Osawa T, Fujioka Y and Noda NN: Structural biology of the core autophagy machinery. Curr Opin Struct Biol 43: $10-17,2017$.

10. Fujikake N, Shin M and Shimizu S: Association between autophagy and neurodegenerative diseases. Front Neurosci 12: $255,2018$.

11. Liu S, Li G, Tang H, Pan R, Wang H, Jin F, Yan X, Xing Y, Chen G, Fu Y and Dong J: Madecassoside ameliorates lipopolysaccharide-induced neurotoxicity in rats by activating the Nrf2-HO-1 pathway. Neurosci Lett 709: 134386, 2019.

12. Fu S, Wang J, Hao C, Dang H and Jiang S: Tetramethylpyrazine ameliorates depression by inhibiting TLR4-NLRP3 inflammasome signal pathway in mice. Psychopharmacology (Berl) 236: 2173-2185, 2019.

13. Ye Q, Zhang Y, Fu J, Zou Y, Zhao W, Chen C and Liu K: Effect of ligustrazine on endometrium injury of thin endometrium rats. Evid Based Complement Alternat Med 2019: 7161906, 2019.

14. Bai XY, Wang XF, Zhang LS, Du PC, Cao Z and Hou Y: Tetramethylpyrazine ameliorates experimental autoimmune encephalomyelitis by modulating the inflammatory response. Biochem Biophys Res Commun 503: 1968-1972, 2018.

15. Lystad AH and Simonsen A: Mechanisms and pathophysiological roles of the ATG8 conjugation machinery. Cells 8: pii: E973, 2019.

16. Kraft LJ, Dowler J, Manral P and Kenworthy AK: Size, organization, and dynamics of soluble SQSTM1 and LC3-SQSTM1 complexes in living cells. Autophagy 12: 1660-1674, 2016.

17. Yamada T, Dawson TM, Yanagawa T, Iijima M and Sesaki H: SQSTM1/p62 promotes mitochondrial ubiquitination independently of PINK1 and PRKN/parkin in mitophagy. Autophagy 15: 2012-2018, 2019.

18. Stjepanovic G, Baskaran S, Lin MG and Hurley JH: Vps34 kinase domain dynamics regulate the autophagic pi 3-kinase complex. Mol Cell 67: 528-534.e523, 2017.

19. Li XT, Liang Z, Wang TT, Yang JW, Ma W, Deng SK, Wang XB, Dai YF, Guo JH and Li LY: Brain-derived neurotrophic factor promotes growth of neurons and neural stem cells possibly by triggering the phosphoinositide 3-kinase/akt/glycogen synthase kinase $3 \beta / \beta$-catenin pathway. CNS Neurol Disord Drug Targets 16: 828-836, 2017.

20. Ishii A, Furusho M, Macklin W and Bansal R: Independent and cooperative roles of the Mek/ERK1/2-MAPK and $\mathrm{PI} 3 \mathrm{~K} / \mathrm{Akt} / \mathrm{mTOR}$ pathways during developmental myelination and in adulthood. Glia 67: 1277-1295, 2019.

21. Gallardo-Vera F, Tapia-Rodriguez M, Diaz D, Fortoul van der Goes T, Montaño LF and Rendón-Huerta EP: Vanadium pentoxide increased PTEN and decreased SHP1 expression in NK-92MI cells, affecting PI3K-AKT-mTOR and Ras-MAPK pathways. J Immunotoxicol 15: 1-11, 2018.

22. Wang H, Liu Y, Wang D, Xu Y, Dong R, Yang Y, Lv Q, Chen X and Zhang Z: The upstream pathway of mtor-mediated autophagy in liver diseases. Cells 8: pii: E1597, 2019. 
23. Fusco C, Mandriani B, Di Rienzo M, Micale L, Malerba N, Cocciadiferro D, Sjøttem E, Augello B, Squeo GM, Pellico MT, et al: TRIM50 regulates Beclin 1 proautophagic activity. Biochim Biophys Acta Mol Cell Res 1865: 908-919, 2018.

24. Kim BW, Jin Y, Kim J, Kim JH, Jung J, Kang S, Kim IY, Kim J, Cheong H and Song HK: The C-terminal region of ATG101 bridges ULK1 and PtdIns3K complex in autophagy initiation. Autophagy 14: 2104-2116, 2018.

25. Caviedes A,Lafourcade C, Soto C and Wyneken U: BDNF/NF- $\mathrm{B}$ signaling in the neurobiology of depression. Curr Pharm Des 23: 3154-3163, 2017

26. Xie XK, Xu ZK, Xu K and Xiao YX: DUSP19 mediates spinal cord injury-induced apoptosis and inflammation in mouse primary microglia cells via the NF-kB signaling pathway. Neurol Res 42: 31-38, 2020
27. Jiang LB, Meng DH, Lee SM, Liu SH, Xu QT, Wang Y and Zhang J: Dihydroartemisinin inhibits catabolism in rat chondrocytes by activating autophagy via inhibition of the NF- $\kappa \mathrm{B}$ pathway. Sci Rep 6: 38979, 2016.

28. Mi B, Wang J, Liu Y, Liu J, Hu L, Panayi AC, Liu G and Zhou W: Icariin activates autophagy via down-regulation of the NF- $\mathrm{BB}$ signaling-mediated apoptosis in chondrocytes. Front Pharmacol 9: 605, 2018

(i) (2) This work is licensed under a Creative Common Attribution-NonCommercial-NoDerivatives 4.0 International (CC BY-NC-ND 4.0) License. 